www.nature.com/pj

\title{
Characterization of toluene and 2-methylnaphthalene transport separated by syndiotactic polystyrene having various crystalline forms
}

\author{
Koichiro Tanigami ${ }^{1,2}$, Daisuke Ishii ${ }^{1}$, Takahiko Nakaoki ${ }^{1}$ and Pieter Stroeve ${ }^{2}$ \\ Diffusion of toluene and 2-methylnaphthalene, in hexane solutions, through amorphous, $\delta_{\mathrm{e}}$ and $\beta$ films of syndiotactic \\ polystyrene (sPS) is investigated. The diffusivity for the transport of toluene (amorphous $>\delta_{\mathrm{e}}$-sPS $>\beta$-sPS) is inversely \\ proportional to the crystallinity. Flux in the non-crystalline region $\left(\bar{N}_{\text {sol }}^{a m}\right)$ for the amorphous and $\delta_{\mathrm{e}}$-sPS films are similar, and \\ greater than for the $\beta$-sPS film, because crystallization is promoted in the amorphous film during transport measurements. \\ Nanopores in crystalline $\boldsymbol{\delta}_{\mathrm{e}}$-sPS act as tunnels for the transport of toluene, whereas there are no such structures for $\boldsymbol{\beta}$-sPS; \\ and thus, the toluene flux is slower. The transport of 2-methynaphthalene is much slower in comparison to toluene, which can \\ be attributed to molecular size. The diffusivity in the amorphous film is slightly larger than that for the $\delta_{\mathrm{e}}$-sPS, whereas no \\ diffusion is observed in the $\beta$-sPS film because of the presence of deformed noncrystalline chains resulting from the high \\ crystallinity. The $\bar{N}_{s o l}^{a m}$ for the amorphous film is larger than that for the $\delta_{\mathrm{e}}$-sPS film, because the pores in the $\boldsymbol{\delta}_{\mathrm{e}}$-sPS are of \\ insufficient size for the transport of 2-methynaphthalene.
}

Polymer Journal (2013) 45, 1135-1139; doi:10.1038/pj.2013.44; published online 8 May 2013

Keywords: diffusivity; 2-methylnaphthalene; nanopore; syndiotactic polystyrene; toluene

\section{INTRODUCTION}

Syndiotactic polystyrene (sPS) is a material with unique properties, including high melting temperature, good chemical resistance and a nanoporous structure in the crystal unit. These properties make it attractive for use as a high-quality engineering thermoplastic. It has been well established that there are five crystalline forms of the molecular structure. The $\alpha$ (trigonal, unit cell $a=b=2.626 \mathrm{~nm}$, $c=0.504 \mathrm{~nm}$ ). ${ }^{1-3}$ and $\beta$ (orthorhombic, unit cell $a=0.881 \mathrm{~nm}$, $b=2.828 \mathrm{~nm}, c=0.551 \mathrm{~nm})^{4,5}$ forms assume an all-trans sequence, whereas the $\gamma$ (uncertain monoclinic suggested, unit cell $a=$ $1.943 \mathrm{~nm}, b=0.851 \mathrm{~nm}, c=0.793 \mathrm{~nm}, \gamma=83.4^{\circ}$ ),$^{6-8} \delta$ (monoclinic, unit cell $\left.a=1.758 \mathrm{~nm}, b=1.326 \mathrm{~nm}, c=0.771 \mathrm{~nm}, \gamma=121.2^{\circ}\right)^{9-12}$ and $\varepsilon$ (orthorhombic, unit cell $a=0.162 \mathrm{~nm}, \quad b=2.20 \mathrm{~nm}$, $c=0.79 \mathrm{~nm})^{13-15}$ forms assume a trans-trans-gauche-gauche (ttgg) sequence. The all-trans conformation is thermally stabilized with a melting temperature as high as $270^{\circ} \mathrm{C}^{1-5} \mathrm{~A}$ crystal with ttgg conformation can be induced by casting from various solutions, including aromatic and chlorinated compounds. The $\delta$ and $\varepsilon$ forms are characterized by the formation of complex structures with organic compounds. These crystals transform to the $\gamma$ form around $130^{\circ} \mathrm{C}^{6-8}$ There are many studies of $\delta$-sPS because solvent molecules can be excluded from the unit cell by soaking in acetone or treating in supercritical $\mathrm{CO}_{2}$, without destroying the crystal. ${ }^{16-19}$ It is worth noting that the nanoporous structure of the crystal is maintained, with the solvent-free form denoted $\delta_{\text {empty }}$-sPS $\left(\delta_{\mathrm{e}}\right.$-sPS). ${ }^{20,21}$ The pore size has been reported to be $115 \AA^{3.21}$ The sorption and desorption of organic solvents in the nanopores of $\delta_{\mathrm{e}}$-sPS were reported by Guerra and colleagues, ${ }^{22,23}$ Tsujita and colleagues ${ }^{24-27}$ and our group. ${ }^{28,29}$ This porous structure is very attractive not only from an academic but also from a practical view, because it is applicable as a sensor for detecting small amounts of organic solvents, adsorption of chemical compounds and so on. Diffusion of organic compounds in sPS can be classified as one of the two processes: transport in the amorphous phase, and transport in the pores of $\delta$-sPS. ${ }^{22}$ Previously, we reported the diffusion of alcohol isomers with different molecular shapes in $\delta_{\mathrm{e}}$-sPS. ${ }^{28,29}$ Linear alcohols diffused the fastest, whereas branched alcohols were much slower, because the molecular size exceeded the pore size.

Transportation of organic compounds in polymer films is an important issue for the selective separation of organic compounds. We previously reported the separation of toluene from a hexane solution by diffusion through syndiotactic polypropylene films, which has four different crystallinities. ${ }^{30}$ As toluene diffuses only in the amorphous phase of syndiotactic polypropylene but not in crystalline phase, the toluene diffusivity depended on the crystallinity.

As an expansion of that work, in this study, amorphous sPS films and films with different crystalline structures were investigated for

${ }^{1}$ Department of Materials Chemistry, Ryukoku University, Otsu, Japan and ${ }^{2}$ Department of Chemical Engineering and Materials Science, University of California, Davis, CA, USA Correspondence: Professor T Nakaoki, Department of Materials Chemistry, Ryukoku University, Seta, Otsu, Shiga 520-2194, Japan.

E-mail: nakaoki@rins.ryukoku.ac.jp

Received 26 February 2013; revised 25 March 2013; accepted 26 March 2013; published online 8 May 2013 
mass transfer of toluene and 2-methylnaphthalene dissolved in hexane solutions. In addition, how the transport properties depend on the size of solvent molecule was compared between toluene and 2-methylnaphthalene.

\section{EXPERIMENTAL PROCEDURE}

\section{Materials}

sPS was purchased from Idemitsu Petrochemical Co Ltd (Tokyo, Japan). The weight average molecular weight and polydispersity index were $2.4 \times 10^{5}$ and 2.3, respectively. Hexane and 2-methylnaphthalene were purchased from Sigma Aldrich (St Louis, MO, USA) and toluene from Fisher Scientific (Pittuburgh, PA, USA).

\section{Film preparation}

Three types of films having different crystalline structures were prepared as follows: melt-quenched sPS film was prepared by quenching in ice water from the melt at $290^{\circ} \mathrm{C}$. Annealed sPS was obtained by gradually cooling to room temperature after melting at $290^{\circ} \mathrm{C}$. Cast sPS was obtained by evaporating the solvent from a $5 \mathrm{wt} \% \mathrm{sPS} /$ chloroform solution and completely excluding the solvent in a vacuum oven for $24 \mathrm{~h}$.

Film thickness was measured using a Mitutoyo Graplate (Mitutoyo Co. Ltd, Kawasaki, Japan). Ten sites were selected, and the thickness was measured at each location. The average of the 10 values, $\bar{L}_{m}$, was adopted as the thickness of the film.

\section{Hexane flow measurements}

Before acquiring the transport measurements, the films were checked for macroscopic pinholes, a very important step in order to determine an accurate diffusion coefficient for an organic solvent in a solid film. The sPS film was placed between two compartments: a hexane reservoir and a sink. A pressure drop of about $3 \mathrm{kPa}$ was applied across the film by raising the hexane tank connected to the upstream compartment with tubing. The flow rate of hexane across the film was measured using a scaled capillary tube connected to the downstream compartment. Films were used in this study if they did not show any hexane flow.

\section{Diffusion experiments}

Diffusion experiments were carried out in a diffusion cell as described in a previous report. ${ }^{30}$ The diffusion cell was separated into two compartments (reservoir and sink) by sPS film. The reservoir was filled with $10 \mathrm{~mm}$ toluene or 2-methylnaphthalene in hexane, while the sink was filled with hexane. The effective area of the film was $2.0 \mathrm{~cm}^{2}$. Both compartments were stirred vigorously in all the diffusion experiments using two magnetic stirrers covered with an adiabatic sheet at room temperature.

\section{Measurements}

$\mathrm{X}$-ray diffraction spectra were carried out using Ni-filtered, $\mathrm{Cu}-\mathrm{K} \alpha$ radiation (40 kV and $200 \mathrm{~mA}$; Rint 2500, Rigaku, Tokyo, Japan). The measurements were carried out from $5^{\circ}$ to $30^{\circ}$, and data were collected at a rate of $2^{\circ} \mathrm{min}^{-1}$, with a scan step of $0.02^{\circ}$. Fourier transform infrared spectra were measured with a resolution of $2 \mathrm{~cm}^{-1}$ (FT-IR-660 Plus, JASCO, Tokyo, Japan). The spectra were measured at room temperature. UV-vis (UV) spectra were measured at room temperature (Cary-3, Varian, Tokyo, Japan).

\section{Absorption coefficient for toluene and 2-methylnaphthalene}

The concentration of toluene or 2-methylnaphthalene transported into the sink compartment was measured via UV spectroscopy. Absorption peaks at 261 and $319 \mathrm{~nm}$ were used for toluene and 2-methynaphthalene, respectively. The concentration of the organic compounds $(c)$ was determined by using the Lambert Beer's law as follows (Equation 1):

$$
A=\varepsilon c l
$$

here $A, \varepsilon$ and $l$ denote the absorbance, absorption coefficient and path length, respectively. The relationship between absorbance and concentration was estimated as follows. The path length was fixed at $1 \mathrm{~mm}$ and the UV spectrum was measured in various concentrations of toluene and 2-methynaphthalene in hexane. Figure 1 shows the calibration curves for toluene and 2-methylnaphthalene. The absorption coefficients for toluene and 2-methynaphthalene were $1.84 \times 10^{6}$ and $2.73 \times 10^{6}$, respectively. From this relationship, the concentration of toluene and 2-methylnaphthalene transferred from the reservoir to the sink was determined.

\section{RESULTS AND DISCUSSION}

\section{Crystal structure and crystallinity}

Figure 2 shows the X-ray diffraction patterns of the annealed, cast and melt-quenched films. Typical diffraction patterns for the $\beta$ - and $\delta_{\mathrm{e}}$ forms were observed for the annealed and cast films, respectively. The melt-quenched film showed a broad diffraction pattern due to the amorphous phase. The degree of crystallinity $\left(X_{c}\right)$ was obtained by calculating the integral intensity of the crystalline peaks $\left(A_{c}\right)$ against the total area of the amorphous and crystalline components $\left(A_{a}+A_{c}\right)$ as shown in Equation 2.

$$
X_{c}=\frac{A_{c}}{A_{a}+A_{c}}
$$

The results are listed in Table 1 . The crystallinity is $33.5 \%$ and $55.7 \%$ for the $\delta_{\mathrm{e}}$ and $\beta$ forms, respectively.

In this study, toluene and 2-methynaphthalene were used for transport measurements in the sPS films. These organic compounds promote crystallization of the $\delta$ form of the crystal, which is featured by the complex structure between the sPS and the solvent molecule. ${ }^{9-12}$ Therefore, the degree of crystallinity was examined both

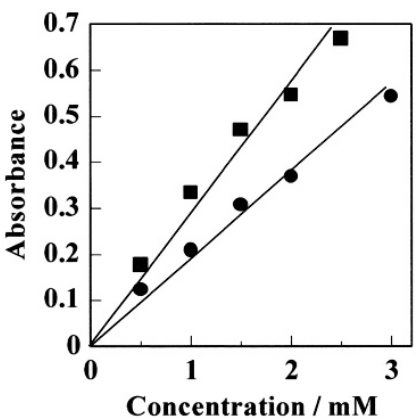

Figure 1 Concentration dependence of absorbance in UV spectra at $261 \mathrm{~nm}$ (-) and $319 \mathrm{~nm}$ ( $\mathbf{\square})$ for toluene and 2-methylnaphthalene in hexane, respectively.

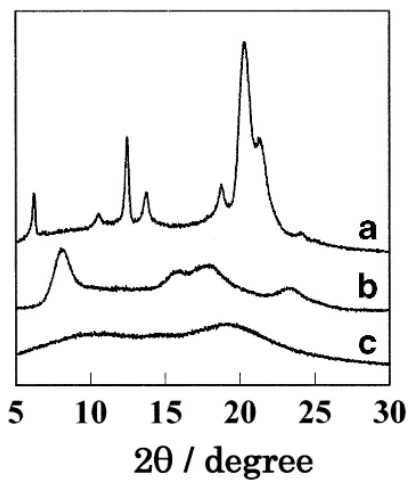

Figure 2 X-ray diffraction patterns of (a) annealed, (b) cast from chloroform and (c) melt-quenched sPS films. These diffraction patterns correspond to the $\beta$-sPS, $\delta_{\mathrm{e}}$-sPS and amorphous sPS films, respectively. 
Table 1 Degree of crystallinity of the amorphous, $\delta_{\mathrm{e}}$-sPS and $\beta$-sPS films before and after the transport measurements, when $\delta$-form crystal formation was induced in the film

\begin{tabular}{lccc} 
& & \multicolumn{2}{c}{ Crystallinity after measurement, \% } \\
\cline { 3 - 4 } & $\begin{array}{c}\text { Crystallinity before } \\
\text { measurement, \% }\end{array}$ & Toluene & 2-Methylnaphthalene \\
\hline B Form & 55.7 & 55.7 & 55.7 \\
Se Form & 33.5 & 38.6 & 35.6 \\
Amorphous & 0 & 30.4 & 13.3 \\
\hline
\end{tabular}

Abbreviation: sPS, syndiotactic polystyrene.

before and after the transport measurement via infrared spectroscopy. Figure 3 shows the infrared spectra of the amorphous, $\delta_{\mathrm{e}}$ - and $\beta$-sPS films before and after the transport measurement. The absorption peak at $572 \mathrm{~cm}^{-1}$ is a very sensitive indicator of the formation of the ttgg crystal. No peak was observed at $572 \mathrm{~cm}^{-1}$ for the amorphous film before the transport measurement, but the regular conformation was confirmed by the appearance of the $572 \mathrm{~cm}^{-1}$ band after the transport measurement for both toluene and 2-methynaphthalene. The peak intensity at $572 \mathrm{~cm}^{-1}$ can be an index of the crystallinity. As estimated by X-ray diffraction measurements, the $\delta_{\mathrm{e}^{-}} \mathrm{sPS}$ film has a crystallinity of $33.5 \%$ with an absorbance of 1.47 at $572 \mathrm{~cm}^{-1}$. Therefore, Equation 3 was obtained relating the crystallinity and the absorbance at $572 \mathrm{~cm}^{-1}$.

$$
X_{C}=\frac{11.6 A_{572}}{l}
$$

Here, $A_{572}$ and $l$ denote the absorbance at $572 \mathrm{~cm}^{-1}$ and the film thickness $(\mu \mathrm{m})$, respectively. The crystallinity after transport measurement is shown in Table 1. After the transport measurements, the crystallinity for the amorphous film became 30.4 and $13.3 \%$ for toluene and 2-methynaphthalene, respectively. Transport of toluene induced a higher crystallinity, close to that of $\delta_{\mathrm{e}}$-sPS film, and the crystallinity induced by 2-methylnaphthalene was much lower. As the molecular size of 2-methylnaphthalene is larger than that of toluene, it is expected that crystallization to the $\delta$ form featuring a complex structure between sPS and 2-methylnaphthalene would be comparatively restricted. The $\delta_{\mathrm{e}^{-}}$and $\beta$-sPS films contain noncrystalline phases, thus it was expected that some of these noncrystalline sPS chains would be converted to $\delta$-form crystals when toluene molecules diffused through the noncrystalline region. However, the spectra acquired after the transport measurements appear almost identical to the original spectra. In fact, the crystallinity of the $\delta_{\mathrm{e}}$-sPS films increased only very slightly from 33.5 to $38.6 \%$ and $35.6 \%$ for toluene and 2-methylnaphthalene, respectively. This shows that further crystallization in the noncrystalline region of the $\delta_{\mathrm{e}}$-sPS film is almost prohibited. For the $\beta$-sPS film, there was no trace of induction of the ttgg conformation for either of the organic compounds, as shown in Figure 3(C2 and C3). This means that the $\delta$ form of the crystal was not induced in the noncrystalline region of the $\beta$-sPS film. These results indicate that the noncrystalline chains in the $\delta_{\mathrm{e}^{-}}$and $\beta$-sPS films are different from those in the amorphous film. Namely, the noncrystalline chains are tightly connected in a three dimensional network structure consisting of crystalline regions as cross-linking points. This prohibits further crystallization.
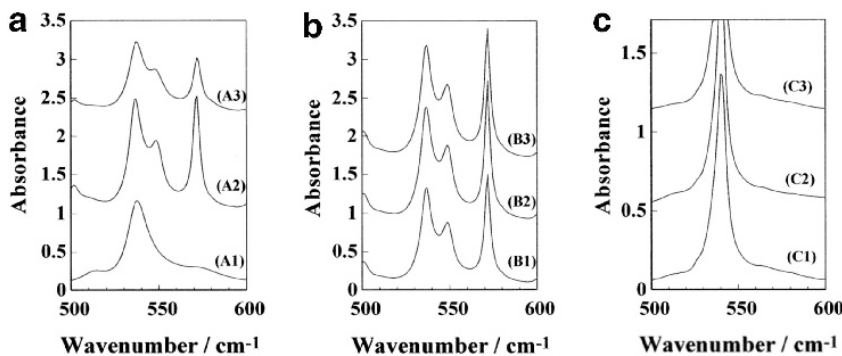

Figure 3 Infrared spectra of the amorphous SPS (a), $\delta_{\mathrm{e}}$-sPS (b) and $\beta$-sPS (c) films before ( $\mathrm{A} 1, \mathrm{~B} 1$ and $\mathrm{C} 1$ ) and after the transport measurements by toluene (A2, B2 and C2) and 2-methynaphthalene (A3, B3 and C3).

\section{Diffusion of toluene in the sPS films}

Transportation of toluene in the sPS films was observed as a function of time via UV spectroscopy. The absorbance was converted to concentration using the calibration curves, and the data are plotted in Figure 4 . The lag time $\theta$, which corresponds to the time that toluene diffuses across the sPS film, is estimated by extrapolating the linear portion of the curve until it intersects the time axis. The lag time for the amorphous sPS film was as short as 2 min. By contrast, the lag time was much longer for the $\delta_{\mathrm{e}^{-}}$and $\beta$-sPS films, whose lag times were 4.0 and $48 \mathrm{~h}$, respectively. The average diffusivity $\bar{D}$ of toluene in the film was estimated using Equation 4:30

$$
\bar{D}=\frac{\bar{L}_{m}^{2}}{6 \theta}
$$

where $\bar{L}_{m}$ is the average film thickness. As the sPS film is swollen by toluene, the film thickness after transport measurement was adopted, because this length corresponds to the actual mass transport thickness. The film thicknesses before and after the transport measurements are shown in Table 2, along with the diffusivity estimated using Equation 4. The diffusion of toluene in the amorphous film was the fastest of these films. This is because there is no hindrance from crystals, although crystallization to the $\delta$ form was promoted after toluene transport. The diffusivity for the $\delta_{\mathrm{e}^{-}}$and $\beta$-sPS films was smaller because of the existence of crystalline regions. As reported in our previous paper concerning toluene transport in syndiotactic polypropylene film, ${ }^{30}$ the lag time strongly depends on the level of crystallinity, and the crystallinities of $\delta_{\mathrm{e}^{-}}$and $\beta$-sPS are 38.6 and $55.7 \%$, respectively. Therefore, the diffusivity of the $\beta$-sPS film was smaller, on the order of $10^{2}$, than that of the $\delta_{\mathrm{e}}$-sPS film.

From the slope of the line in the range $t>\theta$ in Figure 4, the average flux of toluene, $\bar{N}_{\text {sol }}$ was calculated using Equation 5:31

$$
\bar{N}_{s o l}=\frac{d C_{s}}{d t} \cdot \frac{V_{s}}{A_{m}}
$$

where $C_{s}, V_{s}$ and $A_{m}$ denote the concentration of toluene in the sink, the volume of the sink and the film area, respectively. The $\bar{N}_{\text {sol }}$ values are listed in Table 2. The amorphous film provided the largest $\bar{N}_{\text {sol }}$, and the flux for the $\beta$-sPS film was the smallest. As toluene diffuses in the noncrystalline region, the flux was estimated to be equal to the transport in the noncrystalline region $\left(\bar{N}_{s o l}^{a m}\right)$ (Equation 6).

$$
\bar{N}_{s o l}^{a m}=\frac{\bar{N}_{s o l}}{1-x_{c}}
$$

These results are also given in Table 2. As the crystallinity after transport measurement was almost the same for the amorphous and $\delta_{\mathrm{e}}$-sPS films, the $\bar{N}_{\text {sol }}^{a m}$ of toluene is also on the same order of magnitude $\left(10^{-10}\right)$. The $\bar{N}_{\text {sol }}^{a m}$ for the $\beta$-sPS film was one order smaller. This can be explained by the following two reasons: one is the 

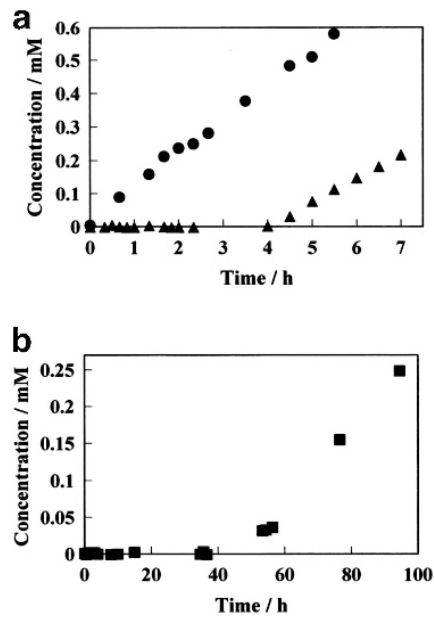

Figure 4 Concentration of toluene in the sink versus time for the amorphous $(\bullet), \delta_{\mathrm{e}}$-sPS $(\mathbf{\Delta})$ and $\beta$-sPS ( $\left.\mathbf{\square}\right)$ films.

Table 2 Diffusion of toluene in the amorphous, $\boldsymbol{\delta}_{\mathrm{e}}$-sPS and $\beta$-sPS films

\begin{tabular}{llllll}
\hline & & & & $\bar{N}_{\text {sol }}$ & $\bar{N}_{\text {sol }}$ \\
& $\theta(\mathrm{h})$ & $\bar{L}_{m}(\mu \mathrm{m})$ & $\bar{D}\left(\mathrm{~cm}^{-2} \mathrm{~s}^{-1}\right)$ & $\left(\mathrm{mol} \mathrm{cm}^{-2} \mathrm{~s}^{-1}\right)$ & $\left(\mathrm{mol} \mathrm{cm}^{-2} \mathrm{~s}^{-1}\right)$ \\
\hline Amorphous & 0.022 & $69.9(61.3)$ & $1.04 \times 10^{-7}$ & $5.16 \times 10^{-10}$ & $7.41 \times 10^{-10}$ \\
$\delta_{\mathrm{e}}$ Form & 4.0 & $98.6(87.0)$ & $1.13 \times 10^{-9}$ & $3.75 \times 10^{-10}$ & $6.11 \times 10^{-10}$ \\
$\beta$ F Form & 48.0 & $40.2(34.1)$ & $1.56 \times 10^{-11}$ & $2.81 \times 10^{-11}$ & $6.39 \times 10^{-11}$ \\
\hline
\end{tabular}

Abbreviation: sPS, syndiotactic polystyrene.

Film thickness is given for the membranes equilibrated with hexane. The values in parenthesis for $\bar{L}_{m}$ show the film thickness before measurement.

differences in the deformed noncrystalline chains of $\delta_{\mathrm{e}^{-}}$and $\beta$-sPS. As the crystallinity of $\beta$-sPS is higher than that of $\delta_{\mathrm{e}}$-sPS, the noncrystalline chains connected by crystalline regions would be more deformed. This reduces the flux of toluene. The other reason involves the transport of toluene in the pores of $\delta_{\mathrm{e}}$-sPS. In fact, the diffusion in the pore of $\delta_{\mathrm{e}}$-sPS has been reported by Guerra and colleagues. ${ }^{22,23}$ The porous structure acts as tunnels for the transport of toluene, which is small enough, allowing for a higher flux of toluene.

\section{Diffusion of 2-methylnaphthalene in sPS films}

Next, the diffusion of 2-methylnaphthalene in hexane across sPS film was investigated. Figure 5 shows the concentration versus time of 2-methylnaphthalene in the sink. The diffusivity is listed in Table 3. The transport of 2-methylnaphthalene is significantly different from that of toluene. In the case of toluene, the lag time in the amorphous sPS film was very fast but that of 2-methylnaphthalene in the amorphous sPS film was as slow as $1.50 \mathrm{~h}$. Similar to toluene, 2methylnaphthalene induced the formation of $\delta$-form crystal in the amorphous film as shown in Figure 3 (A3). However, the peak intensity at $572 \mathrm{~cm}^{-1}$ was not as strong as that of $\delta_{\mathrm{e}}$-sPS. The crystallinity was as low as $13.3 \%$. This is because the bulkiness of 2-methylnaphthalene makes it difficult to form the complex structure with sPS. In addition, the free volume in the amorphous chains of sPS is too small for 2-methylnaphthalene transport. Dammert et al..$^{31}$ estimated that the free volume of amorphous sPS is about $110 \AA^{3}$. The volumetric sizes of single, molecules of toluene and 2-methylnaphthalene are 177 and $237 \AA^{3}$, respectively. Because the film is swollen,
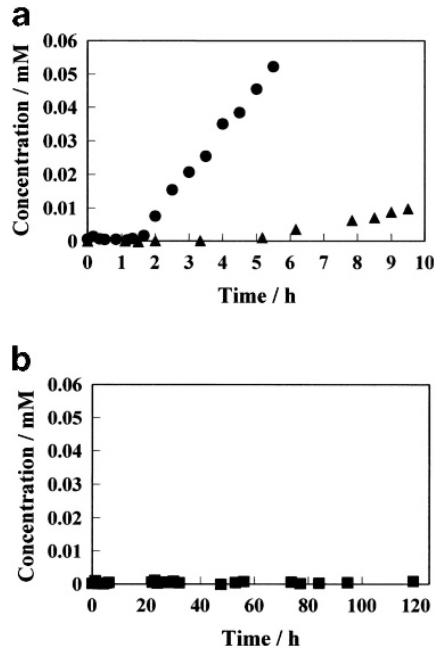

Figure 5 Concentration of 2-methynaphthalene in the sink versus time for the amorphous $(\boldsymbol{O}), \delta_{\mathrm{e}}$-sPS $(\boldsymbol{\Delta})$ and $\beta$-sPS ( $\left.\boldsymbol{\square}\right)$ films.

Table 3 Diffusion of 2-methylnaphthalene in the amorphous and $\delta_{\mathrm{e}}$-sPS films

\begin{tabular}{|c|c|c|c|c|c|}
\hline & $\theta(h)$ & $\bar{L}_{m}(\mu m)$ & $\bar{D}\left(c m^{2} s^{-1}\right)$ & $\begin{array}{c}\bar{N}_{\mathrm{sol}}\left(\mathrm{mol} \mathrm{cm}^{-2}\right. \\
\left.\mathrm{s}^{-1}\right)\end{array}$ & $\begin{array}{c}N_{s o l}{ }^{a m} /\left(1-x_{C}\right) \\
\left(m o l \mathrm{~cm}^{-2} s^{-1}\right)\end{array}$ \\
\hline Amorphous & s 1.50 & $52.2(44.4$ & $8.41 \times 10^{-10}$ & $6.73 \times 10^{-11}$ & $7.76 \times 10^{-11}$ \\
\hline$\delta_{\mathrm{e}}$ Form & 4.53 & $70.1(60.0$ & $5.02 \times 10^{-10}$ & $1.01 \times 10^{-11}$ & $1.57 \times 10^{-11}$ \\
\hline
\end{tabular}

Abbreviation: SPS, syndiotactic polystyrene.

Film thickness is given for the membranes equilibrated with hexane. The values in parenthesis for $\bar{L}_{m}$ show the film thickness before measurement.

the free volume would be expected to be larger, and thus, toluene would be easier to transport in the free volume of sPS, but transport of 2-methylnaphthalene would be more difficult. Therefore, the relationship between the molecular size of 2-methylnaphthalene and the free volume of sPS resulted in slow diffusion, in contrast to the diffusivity of toluene.

The diffusivity in the $\delta_{\mathrm{e}}$-sPS film was slower than in the amorphous film. The crystallinity of $\delta_{\mathrm{e}}$-sPS film is much higher than that induced in the amorphous film after the transport measurements. This results in smaller diffusion in the $\delta_{\mathrm{e}}$-sPS film. It is worth noting that no transport of 2-methylnaphthalene was observed for the $\beta$-sPS film even after $120 \mathrm{~h}$. The infrared spectrum in Figure 3 (C3) shows that the noncrystalline chains in the $\beta$-sPS film were not induced to form the $\delta$-form crystal. Therefore, the lack of transport can be explained by the molecular size of 2-methylnaphthalene. The free volume of the noncrystalline region of the $\beta$-sPS film would not have enough space to accommodate 2-methylnaphthalene diffusion.

The $\bar{N}_{\text {sol }}$ and $\bar{N}_{\text {sol }}^{a m}$ of 2-methynaphthalene are listed in Table 3. The $\bar{N}_{s o l}^{a m}$ for the amorphous film after inducing $\delta$-form crystal was about five times larger than that for the $\delta_{\mathrm{e}}$-sPS film. The newly crystallized $\delta$-sPS contains 2-methylnaphthalene in the unit cell, and this space is large enough for other 2-methylnaphthalene molecules to transport through the pore. In other words, the pore structure acts as a tunnel for the transportation of 2-methylnaphthalene. These large pores are maintained during transport measurement. This makes it possible for 2-methylnaphthalene to transport through the pore. 
However, the pores in $\delta_{\mathrm{e}}$-sPS film are small after excluding m-xylene not large enough for 2-methylnaphthalene transport, and this lack of transport through the pores of the $\delta_{\mathrm{e}}$-sPS crystal results in a smaller flux.

\section{CONCLUSIONS}

Diffusion of hexane solutions of toluene and 2-methylnaphthalene are investigated for amorphous, $\delta_{\mathrm{e}^{-}}$and $\beta$-sPS. Crystalline films of $\delta_{\mathrm{e}^{-}}$-sPS and $\beta$-sPS are characterized by ttgg conformations with nanopores in the unit cell and all-trans conformations. After the transport measurements, $\delta$-sPS crystallization is induced in the amorphous film by both toluene and 2-methylnaphthalene, but crystalline films such as $\delta_{\mathrm{e}}$-sPS and $\beta$-sPS show very little further crystallization. The diffusion of toluene through the amorphous film is the fastest of the three films. This is because there is a noncrystalline region lacking the mass transfer hindrance of the crystalline region. The solute diffusivity in the $\delta_{\mathrm{e}}$-sPS and $\beta$-sPS films depends on the amount of crystallinity. The $\beta$-sPS shows the smallest diffusivity of the three films because of its high crystallinity. The $\bar{N}_{\text {sol }}^{a m}$ of toluene for the amorphous and $\delta_{\mathrm{e}}$-sPS films is almost the same. Due to tolueneinduced formation of the $\delta$-crystal in the amorphous film, the crystallinity after transport measurement is almost the same as for the $\delta_{\mathrm{e}}$-sPS film. This results in similar values of $\bar{N}_{\text {sol }}^{a m}$. The $\bar{N}_{\text {sol }}^{a m}$ of toluene for the $\beta$-sPS film is one order of magnitude smaller than those of the amorphous and $\delta_{\mathrm{e}}$-sPS films. The results can be explained by the transport of solute through the pores of $\delta_{\mathrm{e}}$-sPS, while there are no pores in the unit cell in $\beta$-sPS.

For 2-methylnaphthalene, the diffusivity and mass flux through the sPS films show almost the same trend as for toluene; however, the values are much smaller. This is because 2-methylnaphthalene is 1.3 times larger than toluene. The diffusivities for the amorphous and $\delta_{\mathrm{e}}$-sPS films were almost the same, but no diffusion is observed for the $\beta$-sPS film, even after $120 \mathrm{~h}$. This is again due to the large molecular size of 2-methylnaphthalene, as well as the deformed noncrystalline chains connected by crystalline regions in $\beta$-sPS. This result can be applicable for the separation of toluene and 2-methylnaphthalene. The $\bar{N}_{\text {sol }}^{a m}$ for the $\delta_{\mathrm{e}}$-sPS is five times smaller than that for the amorphous film. The pore size in $\delta_{\mathrm{e}}$-sPS is not large enough for 2-methylnaphthalene transport, but the $\delta$-sPS induced in the amorphous film contains 2-methylnaphthalene in the unit cell, and this space can be used for the transport of 2-methylnaphthalene.

\section{ACKNOWLEDGEMENTS}

This project was carried out under the exchange student program between Ryukoku University, Japan and the University of California Davis, USA. This study was financially supported, in part, by Ryukoku University, Japan.

1 De Rosa, C., Guerra, G., Petraccone, V. \& Corradini, P. Crystal structure of the $\alpha$-form of syndiotactic polystyrene. Polym. J. 23, 1435-1442 (1991).

2 De Rosa, C. Crystal structure of the trigonal modification ( $\alpha$ form) of syndiotactic polystyrene. Macromolecules 29, 8460-8465 (1996).

3 Cartier, L., Okihara, T. \& Lotz, B. The $\alpha$ superstructure of syndiotactic polystyrene:a frustrated structure. Macromolecules 31, 3303-3310 (1998).

4 De Rosa, C., Rapacciuolo, M., Guerra, G., Petraccone, V. \& Corradini, P. On the crystal structure of the orthorhombic form of syndiotactic polystyrene. Polymer 33, 1423-1428 (1992)

5 Chatani, Y., Shimane, Y., Ijitsu, T. \& Yukinari, T. Structural study on syndiotactic polystyrene: 3. Crystal structure of planar form I. Polymer 34, 1625-1629 (1993).
6 Tamai, Y. \& Fukui, M. Thermally induced phase transition of crystalline syndiotactic polystyrene studied by molecular dynamics simulation. Macromolecular Rapid Commun 23, 891-895 (2002).

7 Rizzo, P., Lamberti, M., Albunia, A. R., Ruiz de Ballesteros, O. \& Guerra, G. Crystalline orientation in syndiotactic polystyrene cast films. Macromolecules 35, 5854-5860 (2002).

8 Rizzo, P., Della Guardia, S. \& Guerra, G. Perpendicular chain axis orientation in s-PS films: achievement by guest-induced clathrate formation and maintenance after transitions toward helical and trans-planar polymorphic forms perpendicular chain axis orientation in s-PS films. Macromolecules 37, 8043-8049 (2004).

9 Chatani, Y., Shimane, Y., Ijitsu, T., Yukinari, T. \& Shikuma, H. Structural study on syndiotactic polystyrene: 2 . Crystal structure of molecular compound with toluene. Polymer 34, 1620-1624 (1993).

10 Vittoria, V., De Candia, F., lanelli, P. \& Immirizi, A. Solvent-induced crystallization of glassy syndiotactic polystyrene. Makromol. Chem. Rapid. Commun. 9, 765-769 (1988).

11 Guerra, G., Musto, P., Karasz, F. E. \& MacKnight, W. J. Fourier transform infrared spectroscopy of the polymorphic forms of syndiotactic polystyrene. Makromol. Chem. 191, 2111-2119 (1990)

12 Kobayashi, M., Nakaoki, T. \& Ishihara, N. Molecular conformation in glasses and gels of syndiotactic and isotactic polystyrenes. Macromolecules 23, 78-83 (1990).

13 Rizzo, P., Daniel, C., De Girolamo Del Mauro, A. \& Guerra, G. New host polymeric framework and related polar guest cocrystals. Chem. Mater. 19, 3864-3866 (2007).

14 Rizzo, P., D'Aniello, C., Girolamo Del Mauro, A. \& Guerra, G. Thermal transitions of $\varepsilon$ crystalline phases of syndiotactic polystyrene. Macromolecules 40, 9470-9474 (2007).

15 Petraccone, V., Ruiz, O., Tarallo, O., Rizzo, P. \& Guerra, G. Nanoporous polymer crystals with cavities and channels. Chem. Mater. 20, 3663-3668 (2008).

16 Manfredi, C., De Rosa, C., Guerra, G., Rapacciuolo, M., Auriemma, F. \& Corradini, P. Structural changes induced by thermal treatments on emptied and filled clathrates of syndiotactic polystyrene. Macromol. Chem. Phys. 196, 2795-2808 (1995).

17 Handa, Y. P., Zhang, Z. \& Wong, B. Effect of compressed $\mathrm{CO}_{2}$ on phase transitions and polymorphism in syndiotactic polystyrene. Macromolecules 30, 8499-8504 (1997).

18 Reverchon, E., Guerra, G. \& Venditto, V. Regeneration of nanoporous crystalline syndiotactic polystyrene by supercritical $\mathrm{CO}_{2}$. J. Appl. Polym. Sci. 74, 2077-2082 (1999).

19 Nakaoki, T., Fukuda, Y., Nakajima, E., Matsuda, T. \& Harada, T. Crystallization condition of glassy syndiotactic polystyrene in supercritical $\mathrm{CO}_{2}$. Polym. J. 35, 430-435 (2003).

20 De Rosa, C., Guerra, G., Petraccone, V. \& Pirrozzi, B. Crystal structure of the emptied clathrate form ( $\delta_{\mathrm{e}}$ form) of syndiotactic polystyrene. Macromolecules $30,4147-4152$ (1997).

21 Milano, G., Venditto, V., Guerra, G., Cavallo, L., Ciambelli, P. \& Sannino, D. Shape and volume of cavities in thermoplastic molecular sieves based on syndiotactic polystyrene. Chem. Mater. 13, 1506-1511 (2001).

22 Guerra, G., Manfredi, C., Musto, P. \& Tavone, S. Guest conformation and diffusion into amorphous and emptied clathrate phases of syndiotactic polystyrene. Macromolecules 31, 1329-1334 (1998).

23 Musto, P., Manzari, M. \& Guerra, G. Isothermal guest desorption from crystalline and amorphous phases of syndiotactic polystyrene. Macromolecules 32, 2770-2776 (1999).

24 Mohri, S., Rani, D. A., Yamamoto, Y., Tsujita, Y. \& Yoshimizu, H. Structure and properties of the mesophase of syndiotactic polystyrene-III. Selective sorption of the mesophase of syndiotactic polystyrene. J. Polym. Sci. Part B Polym. Phys. 42, 238-245 (2004)

25 Mahesh, K. P. O., Sivakumar, M., Yamamoto, Y., Tsujita, Y., Yoshimizu, H. \& Okamoto, S. Structure and properties of the mesophase of syndiotactic polystyrene. VIII. Solvent sorption behavior of syndiotactic polystyrene/p-chlorotoluene mesophase membranes. J. Polym. Sci. Part B Polym. Phys. 42, 3439-3446 (2004).

26 Sivakumar, M., Mahesh, K. P. O., Yamamoto, Y., Yoshimizu, H. \& Tsujita, Y. Structure and properties of the $\delta$-form and mesophase of syndiotactic polystyrene membranes prepared from different organic solvents. J. Polym. Sci. Part B Polym. Phys. 43, 1873-1880 (2005).

27 Mahesh, K. P. O., Tsujita, Y., Yoshimizu, H., Okamoto, S. \& Mohan, D. J. Study on $\delta$-form complex in syndiotactic polystyrene-organic molecules systems. IV. Formation of complexes with a mixture of solvents and structural changes during the sorption of solvents by syndiotactic polystyrene mesophase membranes. J. Polym. Sci. Part B Polym. Phys. 43, 2380-2387 (2005).

28 Nakaoki, T., Goto, N. \& Saito, K. Selective sorption and desorption of organic solvent for $\delta$-syndiotactic polystyrene. Polym. J. 41, 214-218 (2009).

29 Furukawa, K. \& Nakaoki, T. Comparison of absorption kinetics of ethanol and butanol into different size nanopores present in syndiotactic polystyrene and poly (p-methylstyrene). Soft Mater. 9, 141-153 (2011).

30 Yamashita, K., Fujiwara, N., Fujikawa, Y., Nakaoki, T., Chiu, W.-Y. \& Stroeve, P. Diffusion and morphological properties of syndiotactic polypropylene (sPP) films. Polym. Eng. Sci. 49, 740-746 (2009).

31 Dammert, R. M., Maunu, S. L., Maurer, F. H. J., Neelov, I. M., Niemelä, S., Sundholm, F. \& Wästlund, C. Free volume and tacticity in polystyrenes. Macromolecules 32, 1930-1938 (1999). 